\title{
Phase Noise Analysis of MEMS-Based Phase Shifters
}

\author{
Gabriel M. Rebeiz
}

\section{Abstract-}

The effect of Brownian noise on Micro-Electro-Mechanical (MEMS)-based circuits has been calculated for MEMS-based circuits (phase shifters, delay circuits). The calculations are done for capacitive shunt MEMS switches and metal-to-metal contact series MEMS switches. The phase noise due to Brownian motion is negligible for MEMS switches with $k \simeq 10 \mathrm{~N} / \mathbf{m}, g_{0}>2 \mu \mathrm{m}, Q>0.5$, and $f_{0} \simeq 50 \mathrm{kHz}$. It is also found that metal-to-metal contact series switches result in much less phase noise than standard capacitive shunt switches. The phase noise increases rapidly for low springconstant bridges $(k=0.2-4 \mathrm{~N} / \mathrm{m})$, low-height bridges, and for bridges with a large mechanical damping $(Q<0.3)$. Also, varactor-based designs result in 30-40 dB more phase noise than switch-based circuits. The paper proves that microwave passive circuits built using MEMS switches (with a proper mechanical design) can be used in most commercial and military applications without any phase noise penalty.

\section{INTRODUCTION}

Micro-electromechanical (MEMS) series and shunt switches have been recently used in many low-loss phase shifters circuits [1], [2], [3], [4], [5], [6]. The MEMS phase shifters are typically implemented using standard PIN diode or FET switch designs, except that the switching element is replaced by a MEMS switch. MEMS switches result in $0.05-0.2 \mathrm{~dB}$ loss at $1-100 \mathrm{GHz}$, and therefore yield excellent performance up to $120 \mathrm{GHz}$. However, MEMS switches suffer from Brownian noise motion which is due to the thermal energy stored in the system. The Brownian noise results in a random change in the capacitance of the switch, which in turn, results in an additional phase and amplitude noise at the output of a MEMS controlled oscillator or phase shifter. The effect of the Brownian noise on oscillators has been derived and demonstrated by Young and Boser [7] and by Dec and Suyama [8], [9]. The goal of this paper is to calculate the Brownian noise effect on phase shifters, and to determine the physical parameters which need to be controlled in the MEMS structure so as to result in very low additional noise.

\section{REVIEW OF BRowniAN NoISE}

The Brownian noise of a mechanical structure with a spring constant, $k$, a damping factor, $b$, and a mechanical resonant frequency, $\omega_{0}$, has been derived by Gabrielson [10] and is:

$$
x_{n}=\frac{\sqrt{4 k_{B} T b}}{k} \frac{1}{1-\left(\omega^{\prime} / \omega_{o}\right)^{2}+j \omega^{\prime} / Q \omega_{o}} \quad \mathrm{~m} / \sqrt{\mathrm{Hz}}
$$

where $k_{B}$ is the Boltzman constant, $Q=k /\left(\omega_{0} b\right)$ is the quality factor of the MEMS bridge, and $\omega_{0}=\sqrt{\mathrm{k} / \mathrm{m}}$ where $m$ is the mass of the bridge. The mechanical force acting on the bridge due to the thermal noise is $f_{n}=$

Gabriel Rebeiz is with the Radiation Laboratory, Department of Electrical Engineering and Computer Science, University of Michigan, Ann Arbor, Michigan, 49109-2122, USA.rebeiz@umich.edu $\sqrt{4 k_{B} T b}$ (in $\mathrm{N} / \sqrt{\mathrm{Hz}}$ ) and is equivalent to the noise voltage from a resistor, $R, v_{n}=\sqrt{4 k_{B} T R}$ (in $\mathrm{V} / \sqrt{\mathrm{Hz}}$ ), with $b$ replaced by $R$. The damping factor, $b$, is dependent on the height of the MEMS bridge, the number of holes in the bridge, and the viscosity of the suspending medium (air, nitrogen, etc.) [10], [11]. The damping factor has also a strong effect on the switching time if the resulting mechanical $Q$ is less than 0.5 . In practice, it is good to design for a $0.5 \geq Q \leq 5$ so as to result in a switching time which is limited by the mechanical characteristics (mass, spring constant, etc.) of the switch and not by the damping of the medium underneath the switch.

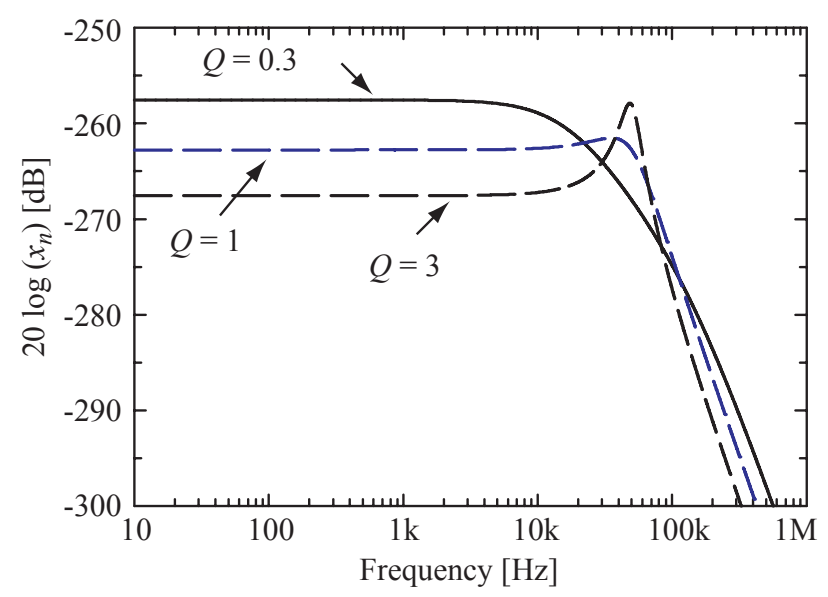

Fig. 1. Calculated Brownian noise component of a MEMS element with $k=10 \mathrm{~N} / \mathrm{m}, f_{0}=50 \mathrm{kHz}, T=300 \mathrm{~K}$, and $Q=0.3,1,3$.

It can be seen that switches with large spring constants and low damping coefficients result in very low Brownian noise. The Brownian noise of a MEMS bridge with $k=$ $10 \mathrm{~N} / \mathrm{m}, f_{0}=50 \mathrm{kHz}, T=300 \mathrm{~K}$, and $Q=0.3,1,3$, are plotted in Fig. 1. If the spring constant is reduced to $1 \mathrm{~N} / \mathrm{m}$, the values on figure 1 will increase by $10 \mathrm{~dB}$. It is seen that the low-frequency value of the Brownian noise is of the order of $10^{-14} \mathrm{~m} / \sqrt{\mathrm{Hz}}$ for a standard MEMS bridge. The Brownian noise decreases very quickly after the mechanical resonant frequency and is insignificant after $5 \omega_{0}$.

The Brownian noise can be represented as a summation of sinusoidal waveforms with random amplitude and phase. A single sinusoid with a mechanical frequency of $\omega^{\prime}$ and amplitude equal to the square root of noise power in a $1 \mathrm{~Hz}$ bandwidth around $\omega^{\prime}$, is written as:

$$
x_{n}=\sqrt{2 \overline{x_{n}^{2}}\left(\omega^{\prime}\right)} \sin \left(\omega^{\prime} t\right) \quad \mathrm{m} / \sqrt{\mathrm{Hz}}
$$

\section{BROWNIAN NOISE EFFECTS FOR MEMS SHUNT SWITCHES}

Consider the MEMS shunt switch shown in Fig. 2. The switch could be the standard capacitive shunt design developed by Raytheon with a center pull-down electrode [2], or 

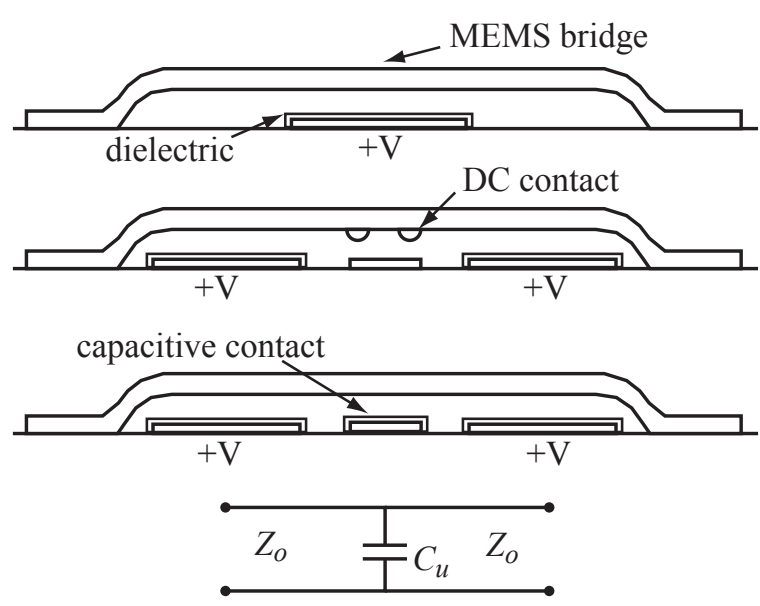

Fig. 2. Different shunt switch topologies (capacitive, DC-contact or capacitive with two-pull down electrodes), all with an up-state capacitance $C_{u}$.

the DC-contact shunt switch with two pull-down electrodes developed by Muldavin et al [12], and Feng et al. [13]. Alternatively, it could also be a low spring-constant switch (Pacheco et al. [4]). What is important is that when the switch is in the up-state position, it allows the microwave energy to pass with very little insertion loss, and when the switch is in the down-state position, it presents a short circuit (or near short circuit) to ground and reflects all the incident energy at the design frequency.

The up-state capacitance of the shunt switch is given by $C_{u}=C_{p p}+C_{f}$, where $C_{f}$ is the fringing capacitance, and $C_{p p}=\epsilon A / g_{0}$ is the parallel-plate capacitance. It is customary to take $C_{f}=\gamma C_{p p}$ and $\gamma<1$ for most designs. When Brownian noise is included, the parallel-plate capacitance can be written as:

$$
C_{p p}=\frac{\epsilon A}{g_{0}+x_{n}} \simeq C_{p p}\left(1-\frac{x_{n}}{g_{0}}\right)
$$

where $x_{n}$ is the Brownian noise movement of the MEMS bridge. The up-state capacitance of the bridge is then:

$$
C_{u}=C_{u 0}\left(1-\frac{1}{1+\gamma} \frac{x_{n}}{g_{0}}\right)
$$

where $C_{u 0}=C_{f}+C_{p p}=(1+\gamma) C_{p p}$ is the up-state capacitance with zero Brownian-noise. The up-state capacitance of the shunt switch results in a scattering parameter of:

$$
S_{21}=\frac{1}{1+\left(j \omega C_{u} Z_{0} / 2\right)}
$$

where $\omega$ is the operating frequency and $Z_{0}$ is the t-line impedance.

\section{A. Phase noise:}

The phase of $S_{21}$ above is the phase delay due to the upstate capacitance of the MEMS switch, and is:

$$
\phi=\frac{-\omega C_{u} Z_{0}}{2}
$$

For $C_{u 0}=35-70 \mathrm{fF}$ and $f=10 \mathrm{GHz}$, the phase delay is $\phi_{0}=0.055-0.11 \mathrm{rad}$. Using Eq. (4), the phase delay with
Brownian noise can be written as:

$$
\phi=\phi_{0}\left(1-\frac{1}{1+\gamma} \frac{x_{n}}{g_{0}}\right)
$$

If a microwave signal with a representation of $A \cos (\omega t)$ is incident on the MEMS bridge, the output signal is (neglecting for now, any amplitude change):

$$
V_{o}(t)=A \cos (\omega t+\phi)=A \cos \left(\omega t+\phi_{0}\left(1-\frac{1}{1+\gamma} \frac{x_{n}}{g_{0}}\right)\right)
$$

Using Eq. (1), the output signal can be expanded to be:

$$
\begin{aligned}
V_{o}(t)= & A \cos \left(\omega t+\phi_{o}\right)+\frac{1}{(1+\gamma)} \frac{A \phi_{o}}{2 g_{o}} \sqrt{2 \overline{x_{n}^{2}}} \\
& \cdot\left[\cos \left[\left(\omega-\omega^{\prime}\right) t+\phi_{o}\right]-\cos \left[\left(\omega+\omega^{\prime}\right) t+\phi_{o}\right]\right]
\end{aligned}
$$

The power in each of the sidebands relative to the carrier is the additional phase noise due to the MEMS bridge, and is:

$$
P_{p h}=\frac{1}{2} \frac{1}{(1+\gamma)^{2}} \frac{\overline{x_{n}^{2}}}{g_{0}^{2}} \phi_{0}^{2} \quad / \mathrm{Hz}
$$

and at low mechanical frequencies $\left(\omega^{\prime}<\omega_{0}\right), \overline{x_{n}^{2}}=$ $\left(4 k_{B} T b\right) / k^{2}=\overline{f_{n}^{2}} / k^{2}$ (in $\mathrm{m}^{2} / \mathrm{Hz}$ ), where $f_{n}$ is the mechanical force due to thermal noise. For $C_{u}=50 \mathrm{fF}$, $\gamma=0.35, f=10 \mathrm{GHz}, Z_{0}=50 \Omega$, and a MEMS bridge parameters given by $k=10 \mathrm{~N} / \mathrm{m}, Q=1, f_{0}=50 \mathrm{kHz}$, and $g_{0}=3 \mu \mathrm{m}$, the phase noise relative to the carrier is calculated to be $P_{p h}=10^{-18}(-180 \mathrm{dBc} / \mathrm{Hz})$ for a single MEMS bridge. If the signal power is $1 \mathrm{~mW}$, the phase noise is much lower than the thermal noise which is $-174 \mathrm{dBm} / \mathrm{Hz}$ (noise figure $=3 \mathrm{~dB}$ ).

There are a four points worth mentioning. The first is the effect of the spring constant on the phase noise $\left(k^{-2}\right)$. If the spring constant drops $1 \mathrm{~N} / \mathrm{m}$, the phase noise increases to $-160 \mathrm{dBc} / \mathrm{Hz}$ for a single MEMS bridge. The second point is the strong dependence of the phase noise on the height of the MEMS structure. Eq. (10) shows that the phase noise depends on the factor $b / g_{0}^{2}$. However, for a fixed-fixed circular or rectangular plate, $b$ depends on $g_{0}^{-3}$ [14], and therefore, the phase noise depends on $g_{0}^{-5}$. If a structure with $g_{0}=3 \mu \mathrm{m}$ is lowered to $g_{0}=1.2 \mu \mathrm{m}$, the phase noise will increase by $20 \mathrm{~dB}$.

The third point is that the phase noise is constant at low offset frequencies, and falls quickly after the mechanical resonant frequency (30-300 kHz, depending on the design). However, for most radar applications with moving target indicators, the final frequencies of interests are in the 5$100 \mathrm{kHz}$ range. Designing a MEMS structure with a low mechanical resonant frequency $(5-20 \mathrm{kHz})$ will not necessarily result in better performance since this structure will generally have a low spring constant and result in a higher low-frequency phase noise. Also, the switching time will be substantially lower in this case.

Finally, Eq. (10) and Eq. (6) seem to indicate that the phase noise is dependent on $f^{2}$. This is not entirely correct for narrowband systems. If a circuit is well designed, then $\phi_{0}=\left(-\omega C_{u 0} Z_{0}\right) / 2$ should be chosen to be the same value at any operation frequency. In other words, $C_{u 0}$ at $f=60 \mathrm{GHz}$ should be $6 \times$ smaller than $C_{u 0}$ at $10 \mathrm{GHz}$. Therefore, the phase noise component is independent of the design frequency. 


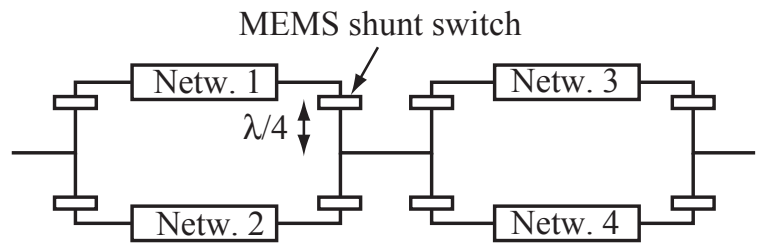

(a)

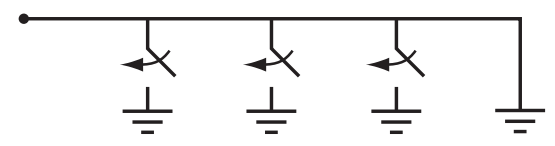

(b)

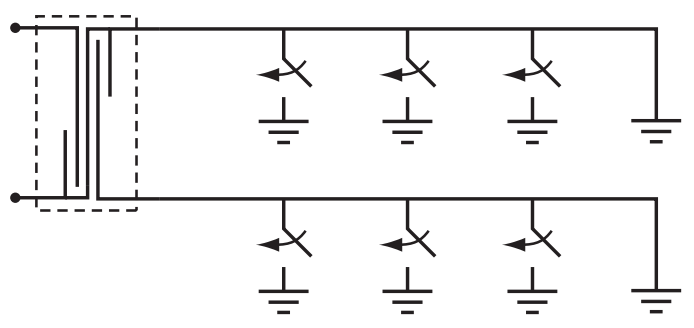

(c)

Fig. 3. A phase shifter based on switched networks (a), switched delay lines (b), and reflect-line with a 3-dB coupler (c).

\section{Phase Shifters based on Shunt Switches}

Phase shifters use a large number of MEMS switches. The Brownian noise of different shunt capacitive switches are not correlated, and therefore, it is easy to prove that phase noise power of multiple shunt switches is the sum of the phase noise powers resulting from each individual switch. This is the same for the total noise in a resistor network. If the MEMS switch is in the up-state position, it contributes a phase noise component to the output signal. However, if the MEMS switch is in the down-state position, it is fixed and does not contribute any phase noise.

Case 1: Switched-Network Designs: A switched network phase shifter requires 4 shunt switches per bit (Fig. 3a), and the signal will always pass by two MEMS switches per bit, depending on the switch positions. For a 3-bit design, the output phase noise is 6 times higher than the phase noise of a single MEMS bridge. If the switched network is a simple transmission line, then it is possible to use 3 switches per bit [15], and the phase noise will be 3 $6 \times$ higher than the phase noise of a single MEMS bridge, depending on the switch selection.

Case 2: Reflect-Line Designs: The phase noise of a reflect-line phase shifter depends on the switch selection. In the case of a delay of $0^{\circ}$, the first set of MEMS switches are pulled down and there is no added phase noise. In the case of the longest delay, the signal passes twice by several MEMS bridges. For a 2-bit design, the longest delay necessitates 3 MEMS bridges and the total phase noise is therefore $6 \times$ higher than the phase noise of a single shunt switch.

Case 3: Reflect-Line with 3-dB Couplers: In this case, the input signal (the carrier) is divided into two parts, each with half of the power and phased $0 / 90^{\circ}$ apart (Fig. 3b) [16]. The phase noise generated in each arm of the $3-\mathrm{dB}$ coupler is the same as Case 2 above, but is referenced to half of the signal power. Also, the phase noise components from each arm of the 3-dB coupler do not add in phase at the output port, and therefore the total noise power from both arms is divided equally between the input and output ports. If the input port is well matched, then the output phase noise relative to the carrier is just half that of Case 2.

If several 3-dB couplers are connected in series to build, for example, a 4-bit phase shifter from two 2-bit phase shifters, then the phase noise at the output port will be the addition of the phase noise resulting from each of the 3-dB coupler/delay-line units. This holds true, of course, if the units are well matched to each other.

\section{BRownian NoIse in MEMS SERIES SWITCHES AND PHASE SHIFTERS}

MEMS series switches do not suffer from Brownian noise in the down-state position since the switch is fixed to the substrate. However, in the up-state position, and if used in a reflection-type phase shifter, MEMS series switches do result in a phase noise component. The reflection phase of a MEMS series switch is:

$$
\phi=-2 \omega C_{u} Z_{0}
$$

and when the value of $C_{u}$ and $x_{n}$ in Eqs. $(2,4)$ are used, the phase noise relative to the carrier due to the reflection from the MEMS series switch becomes:

$$
P_{p h}=\frac{1}{2} \frac{1}{(1+\gamma)^{2}} \frac{\overline{x_{n}^{2}}}{g_{0}^{2}} \phi_{0}^{2} \quad / \mathrm{Hz}
$$

Notice that $C_{u}=2-6 \mathrm{fF}$ in most DC-contact switches, and $\gamma=2-3$ since a large portion of the up-state capacitance of a MEMS series switch is due to the parasitic capacitance between the t-lines and not to the parallel-plate capacitance above the contact points.

MEMS series switches are used in many different phase shifter designs, such as 1) switched t-lines [17], 2) reflection type or 3 ) reflection type with $3-\mathrm{dB}$ couplers. The switched t- line designs result in insignificant phase noise since the MEMS switches must be in the closed position to pass the energy in the different delay sections. Most MEMS series switches, even if based on cantilever designs, have $k>5 \mathrm{~N} / \mathrm{m}$ and $g_{0}>1.5 \mu \mathrm{m}$. The phase noise of a MEMS series switch (in reflection mode) is therefore 100300 times less than that of a shunt capacitance switch (in transmission mode).

The effect of the acceleration noise, acoustic noise and bias voltage noise will also be presented at the conference. Also, we will present the effect of Brownian noise on MEMS varactors and their use in MEMS-based phase shifters.

\section{REFERENCES}

[1] R.E. Mihailovich, M. Kim, J.B. Hacker, E.A. Sovero, J. Studer, J.A Higgins, and J.F. DeNatale, "MEM relay for reconfigurable RF circuits," To Be Published in IEEE Microwave and Wireless Components Letters, Feb 2001.

[2] C.L. Goldsmith, Z. Yao, S. Eshelman, and D. Denniston, "Performance of low-loss RF MEMS capacitive switches," IEEE Microwave Guided Wave Lett., vol. 8, pp. 269-271, August 1998. 
[3] J.B. Muldavin and G.M. Rebeiz, "High isolation MEMS shunt switches; part 1: Modeling," IEEE Trans. on Microwave Theory and Techniques, vol. 48, no. 6, pp. 1045-1052, June 2000.

[4] D. Peroulis, S. Pacheco, and L.P.B. Katehi, "MEMS devices for high isolation switching and tunable filtering," in 2000 IEEE MTT-S Int. Microwave Symp. Dig., Boston, MA, June 2000, pp. 1217-1220.

[5] D. Hyman, A. Schmitz, B. Warneke, T. Y. Hsu, J. Lam, J. Brown, J. Schaffner, A. Walston, R. Y. Loo, G. L. Tangonan, M. Mehregany, and J. Lee, "Surface micromachined RF MEMS switches on GaAs substrates," Int. J. RF Microwave CAE, vol. 9, pp. 348-361, August 1999.

[6] J. Rizk, G.L. Tan, J.B. Muldavin, and G.M. Rebeiz, "High isolation W-band MEMS switches," To be published in IEEE Microwave Wireless Components Letters, vol. 11, no. 1, January 2001.

[7] D. Young and B. Boser, "A micromachined-based RF low-noise voltage controlled oscillator," in IEEE Proc. CICC, May 1997, pp. 431-434.

[8] A. Dec and K. Suyama, "Micromachined electromechanically tunable capacitors and their applications to RF ICs," IEEE Trans. Microwave Theory Techn., vol. 46, no. 12, pp. 2587-2595, Dec. 1998.

[9] A. Dec and K. Suyama, "Microwave MEMS-based voltage controlled oscillators," IEEE Trans. Microwave Theory Techn., vol. 48, no. 11, pp. 1943-1949, Nov. 2000.

[10] T. Gabrielson, "Mechanical-thermal noise in micromachined acoustic and vibration sensors," IEEE Trans. Electron Devices, vol. ED40, pp. 903-909, May 1993.

[11] R. T. Howe and R. S. Muller, "Resonant-microbridge vapor sensor," IEEE Trans. on Electron Devices, vol. ED-33, no. 4, pp. 499-506, April 1986.

[12] J. B. Muldavin and G. M. Rebeiz, "All-metal series and series/shunt MEMS switches," Submitted to IEEE Microwave and Wireless Components Letters, Mar 2001.

[13] S-C Shen and M. Feng, "Low actuation voltage RF MEMS switches with signal frequencies from $0.25 \mathrm{GHz}$ to $40 \mathrm{GHz}$," in IEEE Int. Electronics Device Meeting Proc., December 1999, pp. 689-692.

[14] K.E. Peterson, "Micromechanical membrane switches on silicon," IEEE Trans. Electron. Devices, vol. 23, no. 4, pp. 376-386, July 1978.

[15] B. Pillans, S. Eshelman, A. Malczewski, J. Ehmke, and C.L. Goldsmith, "Ka-band RF MEMS phase shifters," IEEE Microwave Guided Wave Lett., vol. 9, pp. 520-522, Dec. 1999.

[16] Malczewski, S. Eshelman, B. Pillans, J. Ehmke, and C.L. Goldsmith, "X-band RF MEMS phase shifters for phased array applications," IEEE Microwave Guided Wave Lett., vol. 9, pp. 517-519, Dec. 1999.

[17] M. Kim, J.B. Hacker, R.E. Mihailovich, and J.F. DeNatale, "A DCto-40 GHz four-bit RF MEMS true-time delay network," IEEE Microwave and Wireless Components Letters, accepted for publication 2001. 